\title{
Correction to: No relevant mechanical leg axis deviation in the frontal and sagittal planes is to be expected after subtrochanteric or supracondylar femoral rotational or derotational osteotomy
}

Andreas Flury $^{1}$ (D) Armando Hoch ${ }^{1} \cdot$ Sandro Hodel $^{1} \cdot$ Florian B. Imhoff $^{1} \cdot$ Sandro F. Fucentese $^{1} \cdot$ Patrick O. Zingg $^{1}$

Published online: 11 February 2022

(c) The Author(s) under exclusive licence to European Society of Sports Traumatology, Knee Surgery, Arthroscopy (ESSKA) 2022

\section{Correction to:}

Knee Surgery, Sports Traumatology, Arthroscopy

https://doi.org/10.1007/s00167-021-06843-x

Authors would like to update the author given namelfamily name and update the revised version of Fig. 4 to their original publication.

The original article has been corrected.

The original article can be found online at https://doi.org/10.1007/ s00167-021-06843-x.

Andreas Flury

andreas.flury@balgrist.ch

1 Department of Orthopaedics, Balgrist University Hospital,

University of Zurich, Forchstrasse 340, 8008 Zurich,

Switzerland 


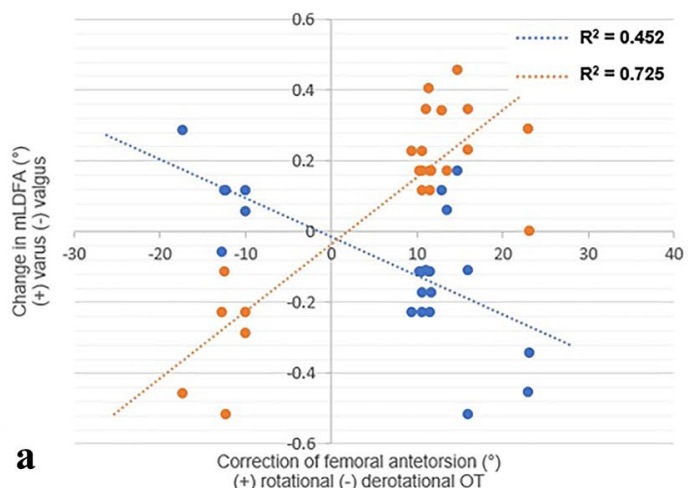

$(+)$ rotational (-) derotational OT

b
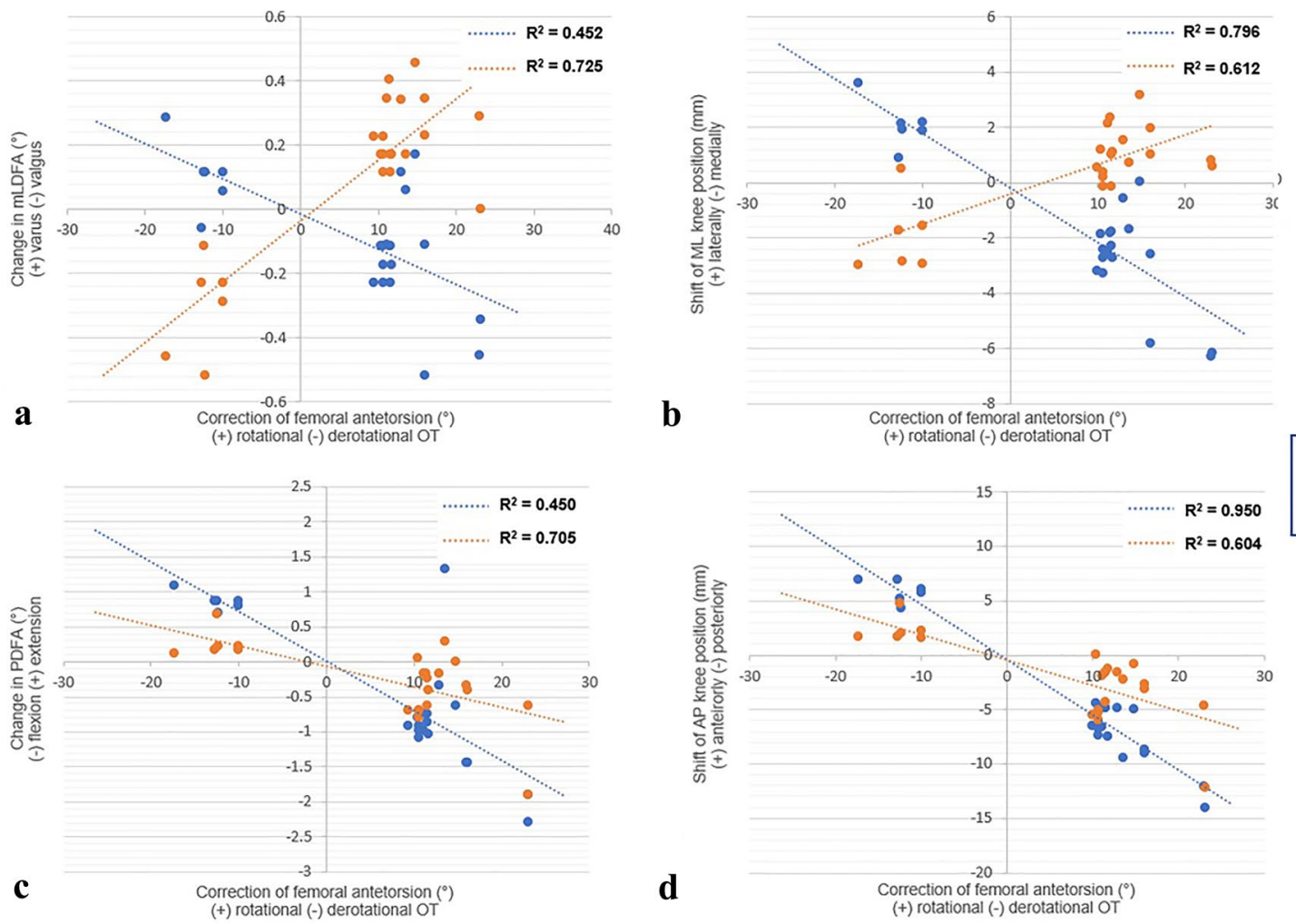

- Subtrochanteric OT - Supracondylar OT

Fig. 4 Influence of femoral rotational/derotational osteotomies on the leg axis in the frontal (A and $\mathbf{B})$ and sagittal $(\mathbf{C}$ and $\mathbf{D})$ planes. Correction of femoral antetorsion correlated linearly to $\mathbf{A}$ change in mLDFA, $\mathbf{B}$ change of medio-lateral knee position, $\mathbf{C}$ change in

PDFA, and D) antero-posterior knee position. Negative values represent the femora derotated due to excessive antetorsion $(n=6)$. Orange and blue dots mark the level of the osteotomy (subtrochanteric versus supracondylar)

Publisher's Note Springer Nature remains neutral with regard to jurisdictional claims in published maps and institutional affiliations. 